\title{
Multimorbidity and Readmissions in Older People with Acute Coronary Syndromes
}

\author{
Gudny Stella Gudnadottir ${ }^{a, b}$, Thorarinn Gudnason ${ }^{d}$ Katarina Wilhelmson ${ }^{a, c}$ \\ Annica Ravn-Fischer ${ }^{e}, f$ \\ aDepartment of Acute Medicine and Geriatrics, Section of Geriatrics, Sahlgrenska University Hospital, Gothenburg, \\ Sweden; 'bchool of Health Sciences, University of Iceland, Reykjavik, Iceland; 'Center for Aging and Health AgeCAP, \\ University of Gothenburg, Gothenburg, Sweden; 'Laeknasetrid, Outpatient Cardiologoy Clinic, Reykjavik, Iceland; \\ eDepartment of Cardiology, Sahlgrenska University Hospital, Gothenburg, Sweden; ${ }^{\prime}$ Institute of Medicine, Department \\ of Molecular and Clinical Medicine, Sahlgrenska Academy, Gothenburg, Sweden
}

\section{Keywords}

Acute coronary syndromes . Over 70 years of age .

Multimorbidity $\cdot$ Readmissions

\begin{abstract}
Aims: This study aimed to examine the multimorbidity as well as the 30-day and 1-year readmission rates in a large, unselected cohort of elderly patients with acute coronary syndrome (ACS). Methods and Results: All patients $\geq 70$ years hospitalized due to ACS during January 1, 2006, to December 31, 2013, and registered in the SWEDEHEART registry were included. In-hospital multimorbidity and disease burden were determined. Outcomes included 30-day and 1-year all-cause mortality, any readmission, and readmissions due to ACS, heart failure, ischaemic stroke or transient ischaemic attack (TIA), and bleeding events. Out of 80,176 patients, 25.6\% had ST-elevation myocardial infarction (STE$\mathrm{MI}$ ) and $74.4 \%$ non-ST-segment elevation ACS (NSTE-ACS). The mean age was 79.8 ( \pm 6.4 standard deviation) and $43.4 \%$
\end{abstract}

Karger@karger.com www.karger.com/crd

Karger $\stackrel{\text { ' }}{5}$

BOPEN ACCESS
(C) 2022 The Author(s)

Published by S. Karger AG, Basel

This article is licensed under the Creative Commons Attribution 4.0 International License (CC BY) (http://www.karger.com/Services/ OpenAccessLicense). Usage, derivative works and distribution are permitted provided that proper credit is given to the author and the original publisher. were women. Multimorbidity, or two chronic diseases, was present in $67.7 \%$, thereof in $53.0 \%$ of STEMI patients and $72.7 \%$ of NSTE-ACS patients. In-hospital mortality was $7.0 \%$. Of the 74,577 patients who survived to discharge, $24.6 \%$ were readmitted within 30 days and $59.5 \%$ were readmitted during the following year. Multimorbid patients had a higher risk of readmissions than those without multimorbidity. Multimorbid STEMI patients were admitted the following year in $56.2 \%$ of cases compared to $44.5 \%$ of STEMI patients without multimorbidity, adjusted odds ratio (OR) 1.35 (95\% confidence interval: 1.26-1.45). Multimorbid patients with NSTE-ACS were readmitted in $63.4 \%$ of cases the following year compared with $49.1 \%$ of those without multimorbidity, adjusted OR 1.42 (1.35-1.50). More than half of the readmissions were due to cardiovascular causes (ACS, stroke, TIA, or heart failure) or bleeding events. Conclusions: Older people with ACS have a high multimorbidity burden and a high readmission rate both within 30 days and 1 year. Half of the readmissions were due to a cardiovascular event or a bleeding event. The presence of multimorbidity increases the risk 
of readmissions for patients with ACS. As hospital admissions are costly for the health care system and can include risks, especially for older patients, there may be opportunities in better risk stratifying this group at discharge for subsequent decrease in readmission rates.

(c) 2022 The Author(s).

Published by S. Karger AG, Basel

\section{Introduction}

Worldwide, the population is ageing, and in a few decades, older patients will be an even more predominant group in health care [1]. Already around $60 \%$ of hospitalizations due to acute coronary syndromes (ACS) are in people 65 years or older [2]. The average age of patients having a myocardial infarction is approximately 76 years for women and 70 years for men in Sweden [3]. With increasing age, more ACS patients will have multimorbidity, defined as at least two chronic diseases. From previous studies, we know that multimorbidity leads to increased risk of readmissions $[4,5]$. Both the European Society of Cardiology and the American Heart Association have recently published consensus guidelines on multimorbidity and other geriatric syndromes in patients with ACS $[6,7]$.

The readmission rate of older people with ACS varies. Previous studies have shown a 30-day readmission rate of $11-35 \%$ [8-10] and a 1-year readmission rate of $40-60 \%$ $[8,10,11]$. The prevalence of multimorbidity varies in different ACS populations and increases with age [12]. In Sweden, all coronary care units register their ACS patients in the national quality registry SWEDEHEART (The Swedish Web-system for enhancement and development of evidence-based care in heart disease evaluated according to recommended therapies). Furthermore, Sweden has a nationwide database for all hospital admissions/readmissions. This gives us a unique chance to examine multimorbidity and readmission rates after all admissions to coronary care units.

The purpose of this article was to determine the multimorbidity and disease burden in an unselected large cohort of older ACS patients, as well as to examine outcome including 30-day and 1-year readmission rates. Readmission could be due to cardiovascular causes and bleeding events, as well as other causes. Since multimorbid as well as older people often are excluded from clinical trials, we think this study, in an unselected and nationwide population, is important to illustrate a major problem - high readmission rates in older multimorbid patients with ACS.

\section{Materials and Methods}

We included all patients who were 70 years or older and hospitalized due to ACS during January 1, 2006, to December 31, 2013, and registered in the SWEDEHEART registry. Only index admissions were included. This age cutoff was chosen because the average age in Sweden for the first myocardial infarction has been approximately 70 years in men and 76 for women, respectively. In previous studies, 70 years of age has been chosen as a cutoff for older people because of mortality inclining after that age [13]. The study was performed with the approval of the Swedish Ethical Review Authority and in accordance with the Declaration of Helsinki.

Primary outcomes were 30-day and 1-year readmissions due to any cause. Secondary outcomes were in-hospital mortality as well as 30-day and 1-year all-cause mortality and readmissions due to ACS, heart failure, ischaemic stroke or TIA, or bleeding events. Results are presented for all ACS patients and for two subgroups: ST-elevation myocardial infarction (STEMI) and non-ST-elevation myocardial infarctions (NSTE-ACS).

Patient characteristics and disease burden were identified from the SWEDEHEART registry and the Swedish National Patient Registry, which collects information about diagnoses at discharge from all hospital stays in Sweden, as well as diagnoses from outpatient hospital specialist care [14]. Outcomes after discharge came from the Swedish National Patient Registry, but episodes of new ACS were also identified from the SWEDEHEART registry. The International Classification of Diseases, 10th Revision (ICD-10), codes for both patient characteristics and outcomes can be found in online supplementary material (for all online suppl. material, see www.karger.com/doi/10.1159/000522016).

The SWEDEHEART registry is a national quality registry in Sweden collecting nationwide data for patients with myocardial infarction admitted to coronary care units. All hospitals providing acute coronary care in Sweden participate in SWEDEHEART, and the registry annually includes 18,000 myocardial infarctions. The registry includes over 100 variables, for example, patients' medical history, strategy before admission, clinical conditions, management during hospital stay, treatment at discharge, and diagnosis. The registry also contains angiographic data, procedural data, and treatment decisions for all patients who are investigated with coronary angiography (CA) and/or revascularized with percutaneous coronary interventions (PCI) at every Swedish centre performing these procedures. To ensure validity of data, 20 randomly selected hospitals are monitored each year and data entered into the SWEDEHEART registry for 40 randomly selected patients compared to patient records [15].

In Sweden, the standard treatment for STEMI is reperfusion therapy with primary PCI. Thrombolysis is only used in the most rural areas (in less than 5\% of STEMI). The standard treatment for NSTE-ACS, in accordance with the guidelines of the European Society of Cardiology, is diagnostic CA with revascularization when appropriate. Both STEMI and NSTE-ACS are treated with acetylsalicylic acid and a $\mathrm{P}_{2} \mathrm{Y}_{12}$ receptor antagonist (together those two are called dual antiplatelet therapy, DAPT) and statins. Most patients have a follow-up after discharge from a specialist nurse or a cardiologist in a cardiology outpatient unit after 6-8 weeks with a second visit after 12-14 months. Many hospitals additionally provide a visit within 2 weeks to a specialist nurse [3]. 


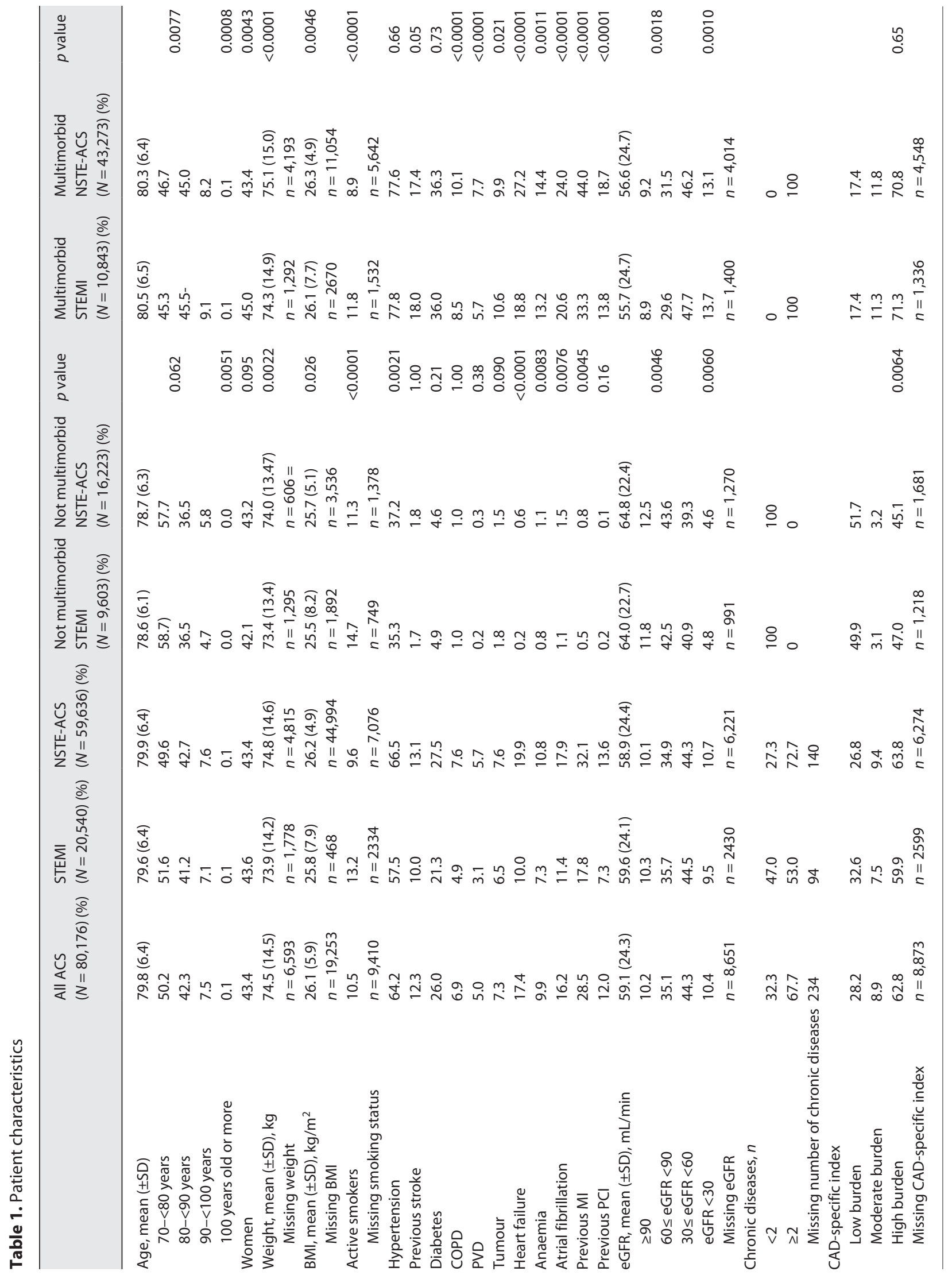




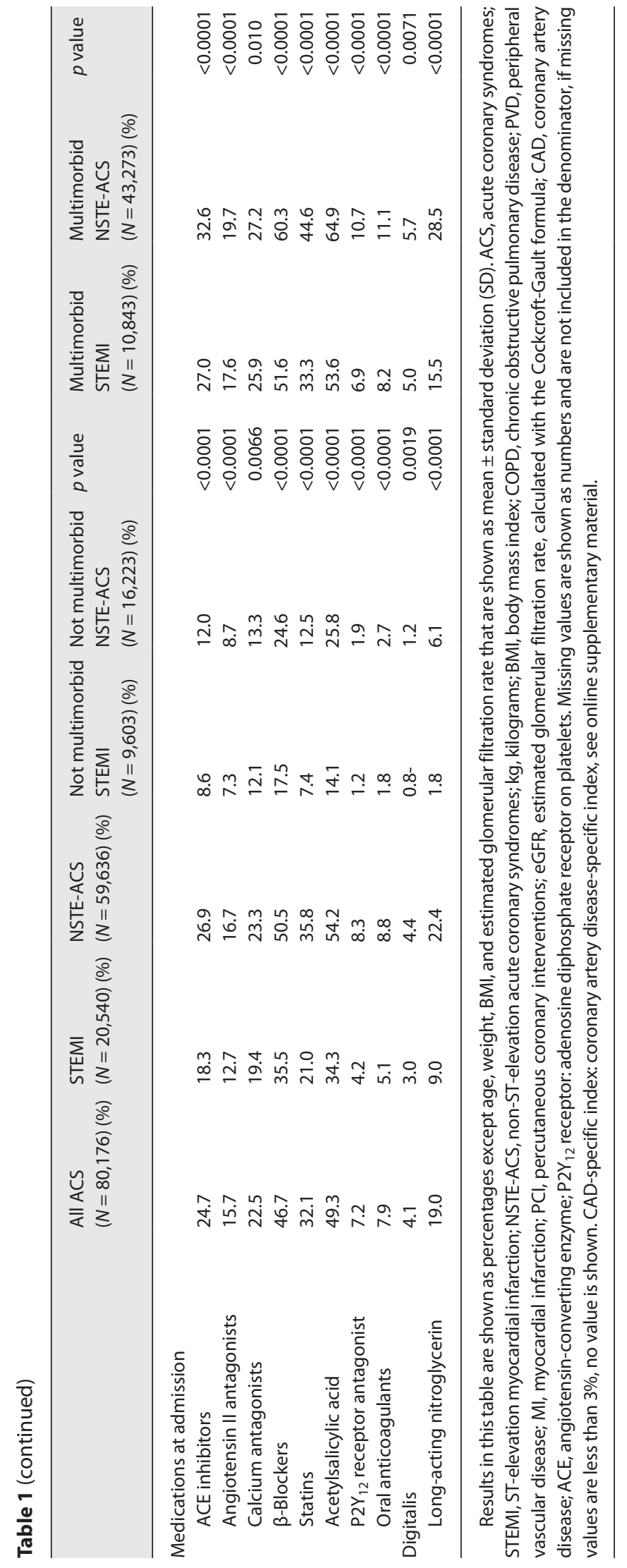

\section{Definitions}

The definition of myocardial infarction used in this article is the same as that used in SWEDEHEART and in all Swedish hospitals; it adheres to the standards of the European Society of Cardiology for definition of myocardial infarctions and ACS [16]. The treating physician makes the final diagnosis. STEMI is defined as the presence of ST-elevation on electrocardiogram (ECG) or new left bundle-branch block on ECG in addition to suspicion of ongoing ischaemia [17]. Renal function was calculated with the Cockcroft-Gault formula and presented as estimated glomerular filtration rate (eGFR). Multimorbidity was defined as two chronic diseases: hypertension, history of stroke or transient ischaemic attacks, previous myocardial infarction, heart failure, atrial fibrillation, peripheral vascular diseases, diabetes, kidney disease, cancer, anaemia, and chronic obstructive pulmonary disease. Multimorbidity burden was measured by the Coronary Artery Disease (CAD)-specific index, allocating patients to low, medium, or high burden giving scores for different comorbidities, as well as smoking status and renal function [18], a description can be found in online supplementary material. Bleeding events were defined as all hospitalizations with a diagnosis of haemorrhage without regard to type or anatomical location, as well as fatal bleeds with the bleeding diagnosis as a first or a second cause of death. ICD-10 codes for bleeding events can be found in online supplementary material, and these codes have previously been used with high sensitivity to identify bleeding events in patients with atrial fibrillation [19].

\section{Statistical Analysis}

Descriptive statistics were employed for the whole group as well as subgroups of STEMI and NSTE-ACS patients with and without multimorbidity; categorical variables were reported as frequency values and proportions, continuous variables with a nearsymmetrical as mean \pm standard deviation (SD), and continuous variables without appropriately near-symmetrical distributions as medians (range). For comparison between groups, Fisher's exact test (lowest 1 -sided $p$ value multiplied by) was used for dichotomous variables. The Mantel-Haenszel $\chi^{2}$ test was used for ordered categorical variables. The $\chi^{2}$ test was used for non-ordered categorical variables, and the $t$ test was used for continuous variables.

To examine the effects of multimorbidity on outcomes, the outcomes for STEMI patients with multimorbidity were compared to those without multimorbidity using multivariate logistic regression adjusting for age, gender, renal disease (eGFR $\geq 90 \mathrm{~mL} / \mathrm{min}$, $60 \leq \mathrm{eGFR}<90 \mathrm{~mL} / \mathrm{min}, 30 \leq \mathrm{eGFR}<60 \mathrm{~mL} / \mathrm{min}$, and $\mathrm{eGFR}<30$ $\mathrm{mL} / \mathrm{min}$ ), heart failure, CAD-specific index, standard risk reduction treatment (treated with acetylsalicylic acid, $\mathrm{P}_{2} \mathrm{Y}_{12}$ receptor antagonists, and statins), and the use of CA (with or without following intervention with PCI or coronary artery bypass grafting $[\mathrm{CABG}])$. Covariates for the multivariate analyses were chosen as they are known predictors of mortality and negative outcomes (5, 9-11). Missing values were handled as an own category in the adjustment. Same calculations were performed for NSTE-ACS patients. Missing variables are shown as numbers in tables if they exceed $3 \%$. 


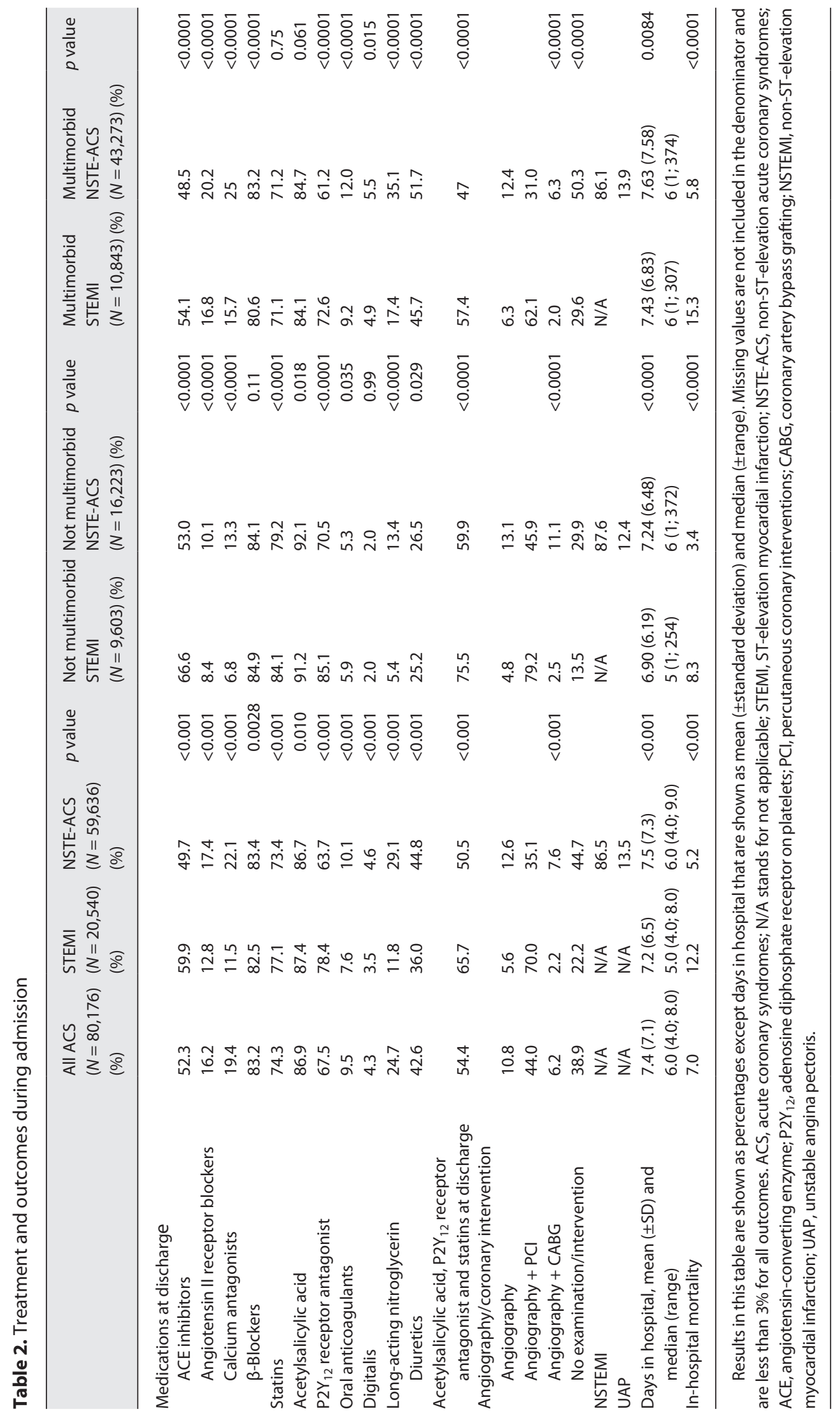




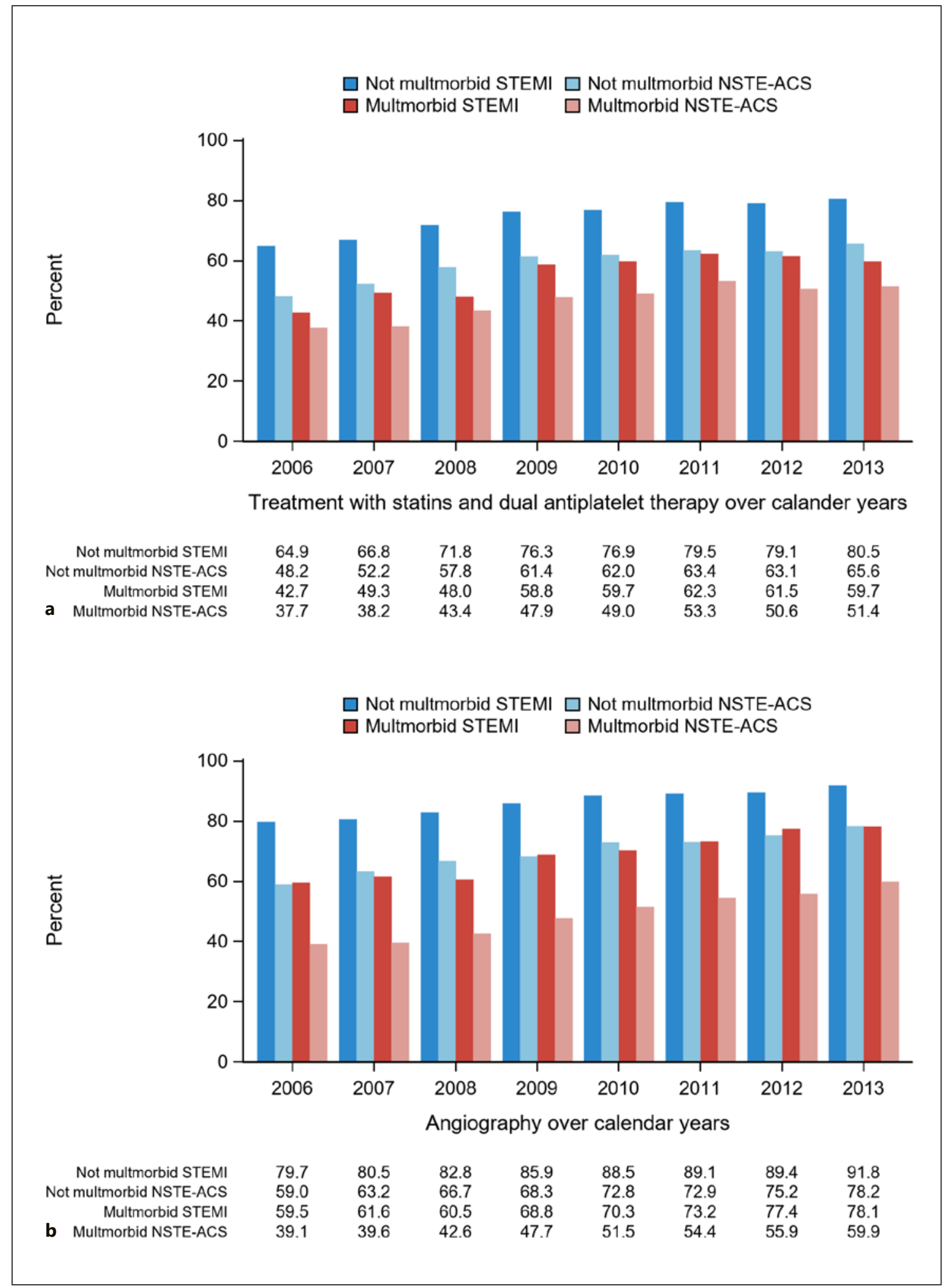

Fig. 1. a, b Treatment with dual antiplatelet therapy together with statins and coronary angiography during 9 years in elderly patients with and without multimorbidity. STEMI, ST-elevation myocardial infarction; NSTEACS, non-ST-elevation acute coronary syndromes. 
Table 3. Outcomes after discharge

\begin{tabular}{llllll}
\hline & Outcomes after discharge & $\begin{array}{l}\text { All ACS } \\
(N=74,577)(\%)\end{array}$ & $\begin{array}{l}\text { STEMI } \\
(N=18,040)(\%)\end{array}$ & $\begin{array}{l}\text { NSTE-ACS } \\
(N=56,537)(\%)\end{array}$ & $p$ value \\
\hline \multirow{2}{*}{30 days } & Total mortality & 3.3 & 3.5 & 3.3 & $<0.0001$ \\
& All-cause readmissions & 25.6 & 21.4 & 25.7 & $<0.0001$ \\
& Readmission due to ACS & 6.4 & 4.8 & 7.0 & $<0.0001$ \\
& Readmission due to stroke or TIA & 0.8 & 0.9 & 0.7 & $<0.0001$ \\
& Readmission due to heart failure & 2.9 & 3.5 & 2.7 & $<0.0001$ \\
& Readmission due to a bleeding event ${ }^{\text {a }}$ & 1.9 & 1.8 & 1.9 & $<0.0001$ \\
& Readmission due to other causes & 13.6 & 10.4 & 13.4 & $<0.0001$ \\
\hline \multirow{2}{*}{ One year } & Total mortality & 14.6 & 12.1 & 15.4 & $<1.5$ \\
& All-cause readmissions & 59.5 & 53.1 & 17.3 & $<0.0001$ \\
& Readmission due to ACS & 15.8 & 11.1 & 3.4 & $<0.001$ \\
& Readmission due to stroke or TIA & 3.4 & 3.3 & 9.6 & $<0.0001$ \\
& Readmission due to heart failure & 9.4 & 8.6 & 6.8 & $<0.0001$ \\
Readmission due to a bleeding event & 6.6 & 23.9 & 24.4 & $<0.0001$ \\
\hline
\end{tabular}

Missing values are not included in the denominator and are less than 3\% for all outcomes; for categorical variables, \% is presented. ACS, acute coronary syndromes; STEMI, ST-elevation myocardial infarction; NSTE-ACS,

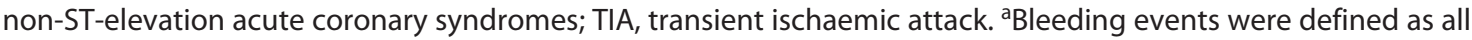
hospitalizations with diagnoses of haemorrhage without regard to type or anatomical location, as well as fatal bleeds with the bleeding diagnosis as a first or a second cause of death. ICD-10 codes for bleeding events can be found in online supplementary material.

\section{Results}

\section{Baseline Variables and Morbidity}

During 2006-2013, 80,176 patients, 70-103 years old, were admitted due to ACS and registered in SWEDEHEART. Of these, 20,540 (25.6\%) had an STEMI and 59,636 had NSTE-ACS (74.4\%). The mean age for all ACS patients was $79.8( \pm 6.4 \mathrm{SD}$ [standard deviation] $)$ and $43.4 \%$ were women. Of the whole group ( 80,176 patients), $26.0 \%$ had diabetes, $17.4 \%$ had heart failure, and $54.7 \%$ had eGFR under $60 \mathrm{~mL} / \mathrm{min} / 1.73 \mathrm{~m}^{2}$. Multimorbidity was present in $67.7 \%$, and the multimorbidity burden according to the CAD-specific index was high in $62.8 \%$ of the patients (Table 1). In the patient group with STEMI, $53.0 \%$ had multimorbidity and $59.9 \%$ had high multimorbidity burden, while of those with NSTE-ACS, 72.7\% had multimorbidity and $63.8 \%$ high multimorbidity burden. In patients without multimorbidity, the multimorbidity burden was considered high for $47.0 \%$ of STEMI patients and $45.1 \%$ of NSTE-ACS patients (Table 1).

\section{Treatments and Investigations}

At discharge, $86.9 \%$ of all ACS patients 70 years and older were treated with acetylsalicylic acid, $67.5 \%$ received a $\mathrm{P} 2 \mathrm{Y}_{12}$ receptor antagonist, and $74.3 \%$ were on statin therapy. The proportion of older patients receiving both DAPT and statins at discharge was 54.4\%. CA with or without a coronary intervention was performed in $61.1 \%$ of the ACS patients, in $77.9 \%$ of STEMI patients and $55.3 \%$ of those with NSTE-ACS (Table 2). In both patients with STEMI and NSTE-ACS, treatment with DAPT together with statins was more common in patients without multimorbidity than those with multimorbidity. In STEMI patients without multimorbidity, 75.5\% were treated with DAPT and statins compared to $57.1 \%$ in the multimorbid group. The corresponding figures for NSTE-ACS patients without multimorbidity treated with DAPT and statins were $59.9 \%$ compared to $47.0 \%$ in those with multimorbidity. CA was performed in most STEMI patients both with and without multimorbidity, $86.5 \%$ and $71.1 \%$ (Table 2). The use of DAPT together with statins as well as diagnostic CA increased in all patient categories during the 9 years the study was performed (shown in Fig. 1).

\section{Mortality and Readmissions}

In-hospital mortality was $7.0 \%$ for all ACS patients, $12.2 \%$ for those with STEMI and 5.2\% for those with NSTE-ACS. Of the 74,577 patients who survived to discharge, $24.6 \%$ were readmitted within 30 days, whereof 


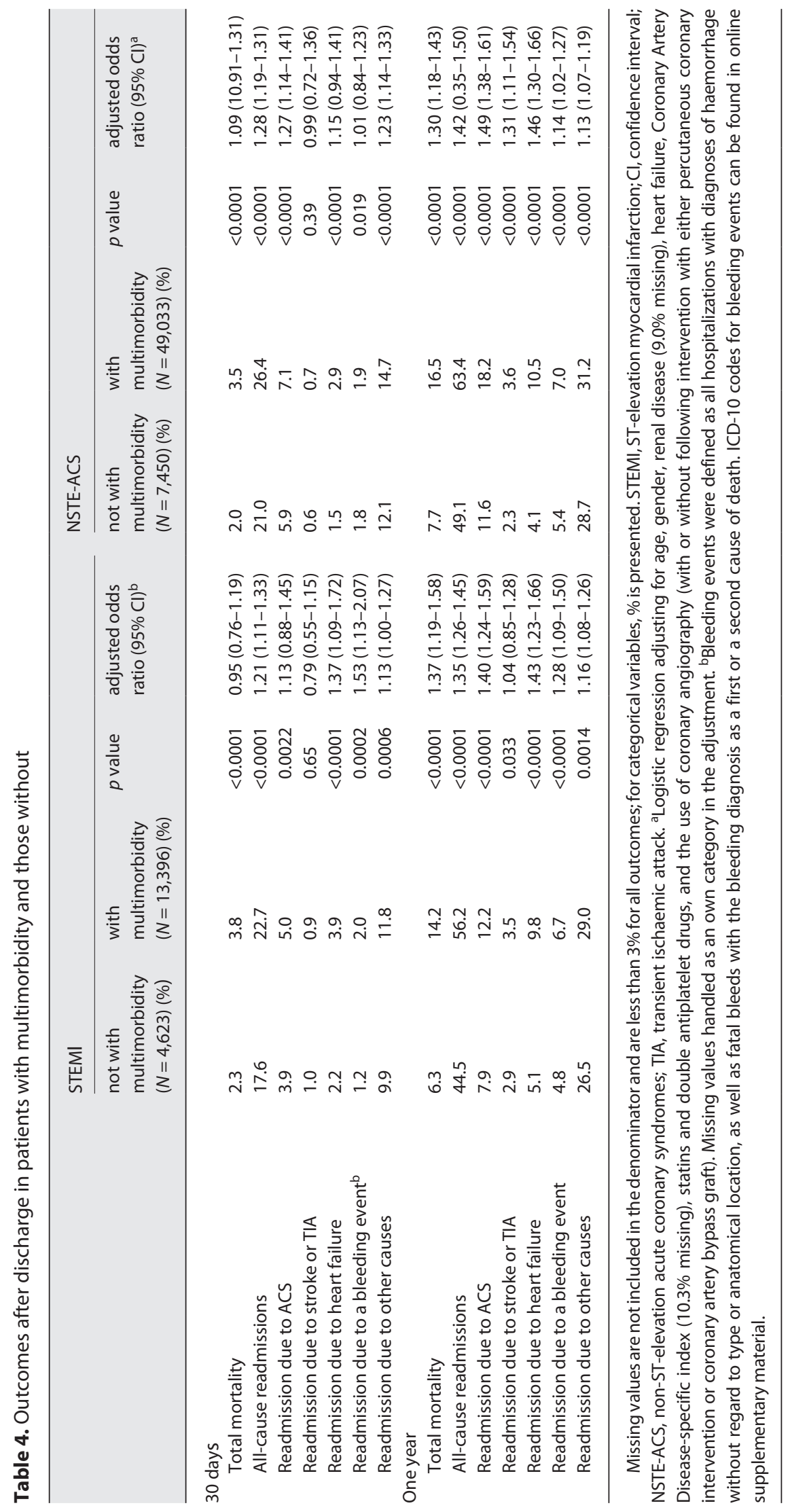


$2.9 \%$ were readmitted within 7 days. In total, $59.5 \%$ of all ACS patients 70 years or older were readmitted within a year from discharge. More than half of those readmissions were due to cardiovascular causes or bleeding events (Table 3). Readmissions rates were higher in the NSTEACS group, $61.5 \%$ versus $53.1 \%$ within 1 year $(p<0.0001)$.

Mortality and readmissions 1 year after discharge were higher in multimorbid patients than in those without multimorbidity. In STEMI patients, the 1-year mortality was $14.2 \%$ versus $6.3 \%$, adjusted odds ratio (OR) 1.37 (95\% confidence interval [CI] 1.19-1.59), and 1-year readmissions were $56.2 \%$ versus $44.5 \%$, adjusted OR 1.35 (1.26-1.45), in those with and without multimorbidity, respectively. In patients with NSTE-ACS, the 1-year mortality after discharge was $16.5 \%$ versus $7.7 \%$, adjusted OR 1.30 (1.18-1.43), and readmissions were $63.4 \%$ versus $49.1 \%$, adjusted OR $1.42(1.35-1.50)$, in those with multimorbidity and those without, respectively (Table 4).

\section{Discussion/Conclusion}

Most people over 70 years old with ACS have multimorbidity and high multimorbidity burden. This is especially prevalent in those with NSTE-ACS where $73 \%$ have multimorbidity and $64 \%$ a high multimorbidity burden. More than half of the patients that survived to discharge were readmitted during the following year, where of almost half of the readmissions were due to cardiovascular causes or bleeding events. Multimorbidity increased the risk of readmissions in patients with STEMI as well as NSTE-ACS. The disease burden in older people is reflected in their treatment as $46 \%$ of all ACS patients did not receive standard therapy with both statins and DAPT, and $22 \%$ of STEMI patients did not undergo CA, while the CA rates in Swedish STEMI patients under 70 years old during the last 15 years has been between 90 and 99\%.

The high proportion of patients with multimorbidity in this older ACS population reflects the high prevalence of multimorbidity in the general older population, as up to $70 \%$ of community-dwelling people over 65 years old have multimorbidity $[12,20]$. The rate of diabetes in older people with ACS has been reported to vary from 15 to $35 \%$, hypertension from 45 to $79 \%$, and anaemia $10-43 \%$ [21-23], in concordance with our results. The prevalence of diabetes in the general population of Swedish 70 year olds in 2005 was $16 \%$, and the prevalence for hypertension was 73\% [24]. Multimorbidity burden was considered high in a considerable proportion of patients without multimorbidity according to the CAD-specific index, as this index takes into consideration renal function and smoking status [18]. The presence of comorbidities can influence the benefit/risk balance of ACS treatment, either through increasing the risk of new ACS or the risk of bleeding events, renal failure, or other complications. Therefore, when treating older people with ACS, all comorbidities must be considered.

The low proportion of older patients receiving standard therapy with DAPT and statins as well as a rather large percentage with STEMI not undergoing CA may have many different causes. Most importantly, the study included patients treated from 2006 to 2013, and during this period, and since, the use of all these therapies has increased. For example, CA in STEMI patients over 80 years old in Swedish Coronary Care units during 19942014 increased from less than $60 \%$ to around $74-82 \%$ (women vs. men) and for those who were 65-79 years old from $50-60 \%$ to over $80-90 \%$ [25] and the use of DAPT in all myocardial infarction patients from $65 \%$ to over $80 \%$ [3]. Half of the patients in this study were over 80 years old. There was an increase in the use of these therapies in Sweden in this age group as well during the study period, for example, statin use from less than $50 \%$ to $80 \%$ in men. Also, concerns about adverse events of these treatments, especially bleeding events, partly explain these results. The annual risk for a major bleeding event in those who are over 85 years old receiving ASA alone is over $4 \%$ [26]. A study in multimorbid older patients in Sweden with ACS showed a readmission rate of $10 \%$ due to bleeding events for 1 year [27]. Aside from the high age of patients in that study, $10 \%$ had anaemia at admission and $55 \%$ had eGFR under $60 \mathrm{~mL} / \mathrm{min}$, all of these are factors that increase the risk of bleeding events [28, 29]. Complications after statin use are also more common in elderly patients, especially those with multimorbidity and polypharmacy [30].

The high readmission rate shown in this study is in concordance with some studies in unselected cohorts of older people with ACS which show the 1-year readmission rate to be up to $60 \%$ and the 30 -day readmission rate to be around $20-35 \%[8,9,11]$. An American study in unselected ACS population found 1-year admissions to be $62 \%$, and half of those were not cardiovascular, results that are almost identical to those we found [10]. The factors that are most associated with readmissions after ACS are age, diabetes, length of stay of 7 days or more, pulmonary disease, peripheral artery disease, renal disease, and heart failure [10, 31]. Multimorbidity is also associated with higher readmission risk and other outcomes as was shown in this study [32]. In addition to being costly, hos- 
pital admissions often cause a loss of function and increased dependency in older people [33]. Therefore, finding ways to reduce readmissions is important. In an ACS population, an early follow-up visit to a nurse 14 days after discharge reduced readmissions [34] supporting that this high readmission rate can be reduced with different methods.

Older patients are a very heterogenous group regarding how heavy the multimorbidity burden is, how frail or fit they are, the prevalence of cognitive impairment, and polypharmacy, as well as different levels of dependency. As patients become more multimorbid, frail, and dependent, the goal of treatment shifts from prolonging life to increasing the quality of life and eventually to palliative care. The multimorbidity and its accompanying polypharmacy becomes problematic as it increases the risk for medication-related side effects of evidence-based treatments for ACS such as statins and ACE inhibitors and antiplatelet drugs. These prescription decisions and clinical decisions are ethically challenging as it can be near impossible to judge the tipping point for more risks than benefits associated with ACS treatments. The 2020 ESC guidelines do state that older patients should receive the same diagnostic as well as revascularization treatments, but the evidence level is category B [35], indicating that not many randomized studies are behind the recommendations. Personalizing care in cooperation with the patients' wishes is important. To improve the quality of care of older people with ACS and reduce readmission rates, it is essential to take multimorbidity and other geriatric syndromes into account when tailoring individual follow-up routines. In the elderly, it is also important to do an accurate risk-benefit analysis of different kinds of treatment. Both the AHA and ESC now recommend this $[6,7,35]$. The risk associated with antiplatelet drugs is partially time related, so the use of bleeding prediction models such as the PRECISE-DAPT score [29] might aid in shortening the length of DAPT treatment in those with the highest bleeding risk. To recognize ACS patients with frailty already in the coronary care unit is also important. With geriatric consultant teams and subacute care at geriatric rehabilitation wards, the frail patients could get important support [6]. After discharge, the frailest and most multimorbid ACS patients might benefit from earlier follow-up than usual, i.e., within 7 days of discharge to decrease readmissions [36].

\section{Strengths and Limitations}

This study provides insights into a large, nationwide, real-life cohort of multimorbid older people with ACS who are often excluded from randomized trials. As the SWEDEHEART Registry is used in all coronary care units in Sweden and the Swedish Patient Registry includes all admissions, this study focuses on the complex aftermath after ACS in older people rather than only the cardiovascular outcomes. The all-inclusive nature of SWEDHEART minimizes selection bias, and this nationwide insight into the quality of care for older people with ACS may help to further improve both treatment and follow-up and may potentially save resources.

The older patients who were not admitted to coronary care units are not included in this study, and among those patients might be the most frail and complex older patients. When using hospital diagnoses to look at the multimorbidity burden, the registries underdiagnose diseases that often do not lead to admission, an example is chronic obstructive pulmonary disease and peripheral vascular disease. The multimorbidity burden of these ACS patients might therefore be even higher than is described.

The most important limitation of this study is that it includes data from more than 7 years ago. However, these are the data we as of now have available to publish. During this time, the implementation of both medical therapy and interventions with CA, PCI, and CABG has changed. However, the readmission rates have not declined from 2013 to 2019 [3], so we believe the data remain both important and relevant. A further unfortunate limitation is that we did not register peptic ulcer disease in the year before admission in this study which is an important factor in the risk for bleeding events.

\section{Acknowledgments}

Aldina Pivodic, statistician at Statistiska Konsult Gruppen in Gothenburg, performed the statistical analyses. All analyses were overseen by the first author who also interpreted the results.

\section{Statement of Ethics}

The study was performed with the approval of the Swedish Ethical Review Authority, Reference No. Dnr 2015/272, and its content complies with the Declaration of Helsinki. As data in the SWEDEHEART registry are collected as part of health care quality improvement, informed consent from participants is not required by the Swedish law, but patients are made aware of the registry and its use and can decline participation.

\section{Conflict of Interest Statement}

The authors have no conflicts of interest to declare. 


\section{Funding Sources}

This work is a part of a doctoral thesis by Gudny Stella Gudnadottir who has received grants from Landspitali-University Hospital Science Fund, The Gothenburg Medical Society, and the Research fund of the University of Iceland.

\section{Author Contributions}

Gudny Stella Gudnadottir is responsible for the idea behind the paper, she is the main contributor to the design of the work, the acquisition, and interpretation of data, and assisted in the analyses for the work. She is also responsible for drafting the manuscript. Thorarinn contributed substantially to the design of the work as well as acquisition and interpretation. Katarina Wilhelmson contributed with drafting of the work as well as interpretation. Annica Ravn-Fischer contributed with the acquisition as well as interpretation of the work. All authors contributed to revising the actual content and have approved the final version to be published and agree to be accountable for all aspects of the work in ensuring that questions related to the accuracy or integrity of any part of the work are appropriately investigated and resolved.

\section{Data Availability Statement}

The data underlying this article cannot be shared publicly due to the privacy of individuals that participated in the study. The data will be shared on reasonable request to the corresponding author.

\section{References}

1 Beard JR, Officer AM, Cassels AK. The world report on ageing and health. Gerontologist. 2016;56(Suppl 2):S163-6.

2 Dai X, Busby-Whitehead J, Alexander KP. Acute coronary syndrome in the older adults. J Geriatr Cardiol. 2016;13(2):101-8.

3 SWEDEHEART Annual Report 2019. 2020. Report No.: ISSN: 2000-1843.

4 Alfredsson J, Alexander KP. Multiple chronic conditions in older adults with acute coronary syndromes. Clin Geriatr Med. 2016;32(2): 291-303.

5 Aubert CE, Schnipper JL, Fankhauser N, Marques-Vidal P, Stirnemann J, Auerbach $\mathrm{AD}$, et al. Patterns of multimorbidity associated with 30-day readmission: a multinational study. BMC Public Health. 2019;19(1):738.

6 Damluji AA, Forman DE, van Diepen S, Alexander KP, Page RL 2nd, Hummel SL, et al. Older adults in the cardiac intensive care unit: factoring geriatric syndromes in the management, prognosis, and process of care - a scientific statement from the American heart association. Circulation. 2020;141(2):e6-e32.

7 Walker DM, Gale CP, Lip G, Martin-Sanchez FJ, McIntyre HF, Mueller C, et al. Frailty and the management of patients with acute cardiovascular disease: a position paper from the acute cardiovascular care association. Eur Heart J. 2018;7:204887261875893.

8 Ephrem G. Red blood cell distribution width is a predictor of readmission in cardiac patients. Clin Cardiol. 2013;36(5):293-9.

9 Dharmarajan K, Hsieh AF, Lin Z, Bueno H, Ross JS, Horwitz LI, et al. Diagnoses and timing of 30-day readmissions after hospitalization for heart failure, acute myocardial infarction, or pneumonia. JAMA. 2013;309(4):35563.

10 Southern DA, Ngo J, Martin BJ, Galbraith PD, Knudtson ML, Ghali WA, et al. Characterizing types of readmission after acute coronary syndrome hospitalization: implications for quality reporting. J Am Heart Assoc. 2014; 3(5):e001046
11 Khumri TM, Reid KJ, Kosiborod M, Spertus JA, Main ML. Usefulness of left ventricular diastolic dysfunction as a predictor of 1-year rehospitalization in survivors of acute myocardial infarction. Am J Cardiol. 2009;103(1): 17-21.

12 Barnett K, Mercer SW, Norbury M, Watt G, Wyke S, Guthrie B. Epidemiology of multimorbidity and implications for health care, research, and medical education: a cross-sectional study. Lancet. 2012;380(9836):37-43.

13 Caterino JM, Valasek T, Werman HA. Identification of an age cutoff for increased mortality in patients with elderly trauma. Am J Emerg Med. 2010;28(2):151-8.

14 Ludvigsson JF, Andersson E, Ekbom A, Feychting M, Kim JL, Reuterwall C, et al. External review and validation of the Swedish national inpatient register. BMC Public Health. 2011;11:450.

15 Jernberg T, Attebring MF, Hambraeus K, Ivert $\mathrm{T}$, James $\mathrm{S}$, Jeppsson $\mathrm{A}$, et al. The Swedish web-system for enhancement and development of evidence-based care in heart disease evaluated according to recommended therapies (SWEDEHEART). Heart. 2010; 96(20):1617-21.

16 Thygesen K, Alpert JS, Jaffe AS, Chaitman BR, Bax JJ, Morrow DA, et al. Fourth universal definition of myocardial infarction (2018). J Am Coll Cardiol. 2018;72(18):2231-64.

17 Thygesen K, Alpert JS, Jaffe AS, Simoons ML, Chaitman BR, White HD, et al. Third universal definition of myocardial infarction. J Am Coll Cardiol. 2012;60(16):1581-98.

18 Sachdev M, Sun JL, Tsiatis A, Nelson CL, Mark DB, Jollis JG. The prognostic importance of comorbidity for mortality in patients with stable coronary artery disease. J Am Coll Cardiol. 2004;43:576-82.

19 Friberg L, Skeppholm M. Usefulness of health registers for detection of bleeding events in outcome studies. Thromb Haemost. 2016; 116(6):1131-9.
20 Rocca WA, Boyd CM, Grossardt BR, Bobo WV, Finney Rutten LJ, Roger VL, et al. Prevalence of multimorbidity in a geographically defined American population: patterns by age, sex, and race/ethnicity. Mayo Clin Proc. 2014;89(10):1336-49.

21 Yeh RW, Sidney S, Chandra M, Sorel M, Selby JV, Go AS. Population trends in the incidence and outcomes of acute myocardial infarction. N Engl J Med. 2010;362(23):2155-65.

22 Radovanovic D, Seifert B, Urban P, Eberli FR, Rickli H, Bertel O, et al. Validity of charlson comorbidity index in patients hospitalised with acute coronary syndrome. Insights from the nationwide AMIS plus registry 20022012. Heart. 2014;100(4):288-94.

23 Stucchi M, Cantoni S, Piccinelli E, Savonitto S, Morici N. Anemia and acute coronary syndrome: current perspectives. Vasc Health Risk Manag. 2018;14:109-18.

24 Zhi X, Joas E, Waern M, Östling S, BörjessonHanson A, Skoog I. Prevalence of cardiovascular disorders and risk factors in two 75-year-old birth cohorts examined in 19761977 and 2005-2006. Aging Clin Exp Res. 2013;25(4):377-83.

25 Szummer K, Wallentin L, Lindhagen L, Alfredsson J, Erlinge D, Held C, et al. Improved outcomes in patients with ST-elevation myocardial infarction during the last 20 years are related to implementation of evidence-based treatments: experiences from the SWEDEHEART registry 1995-2014. Eur Heart J. 2017;38(41):3056-65.

26 Li L, Geraghty OC, Mehta Z, Rothwell PM. Age-specific risks, severity, time course, and outcome of bleeding on long-term antiplatelet treatment after vascular events: a population-based cohort study. Lancet. 2017.

27 Gudnadottir GS, James SK, Andersen K, Lagerqvist B, Thrainsdottir IS, Ravn-Fischer A, et al. Outcomes after STEMI in old multimorbid patients with complex health needs and the effect of invasive management. Am Heart J. 2019;211:11-21. 
28 Melloni C, Cornel JH, Hafley G, Neely ML, Clemmensen $\mathrm{P}$, Zamoryakhin D, et al. Impact of chronic kidney disease on long-term ischemic and bleeding outcomes in medically managed patients with acute coronary syndromes: Insights from the TRILOGY ACS Trial. Eur Heart J Acute Cardiovasc Care. 2016;5(6):443-54.

29 Costa F, van Klaveren D, James S, Heg D, Raber L, Feres F, et al. Derivation and validation of the predicting bleeding complications in patients undergoing stent implantation and subsequent dual antiplatelet therapy (PRECISE-DAPT) score: a pooled analysis of individual-patient datasets from clinical trials. Lancet. 2017;389(10073):1025-34.

30 Armitage J. The safety of statins in clinical practice. Lancet. 2007;370(9601):1781-90.
31 Kwok CS, Narain A, Pacha HM, Lo TS, Holroyd EW, Alraies $\mathrm{MC}$, et al. Readmissions to hospital after percutaneous coronary intervention: a systematic review and meta-analysis of factors associated with readmissions. Cardiovasc Revasc Med. 2019.

32 Kociol RD, Peterson ED, Hammill BG, Flynn KE, Heidenreich PA, Pina IL, et al. National survey of hospital strategies to reduce heart failure readmissions: findings from the get with the guidelines-heart failure registry. Circ Heart Fail. 2012;5(6):680-7.

33 Covinsky KE, Palmer RM, Fortinsky RH, Counsell SR, Stewart AL, Kresevic D, et al. Loss of independence in activities of daily living in older adults hospitalized with medical illnesses: increased vulnerability with age. J Am Geriatr Soc. 2003;51(4):451-8.
34 Bumpus SM, Krallman R, Kline-Rogers E, Montgomery D, Eagle KA, Rubenfire M. Transitional care to reduce cardiac readmissions: 5-year results from the BRIDGE clinic. J Fam Med Dis Prev. 2017;3(3):1-7.

35 Collet JP, Thiele H, Barbato E, Barthélémy $\mathrm{O}$, Bauersachs J, Bhatt DL, et al. ESC Guidelines for the management of acute coronary syndromes in patients presenting without persistent ST-segment elevation. Eur Heart J. 2020; 42(14):1289-367.

36 Jackson C, Shahsahebi M, Wedlake T, DuBard CA. Timeliness of outpatient follow-up: an evidence-based approach for planning after hospital discharge. Ann Fam Med. 2015; 13(2):115-22. 